\title{
Identify human cluster of differentiation 147 (CD147), a new target of SARS-CoV-2 invasion
}

\begin{abstract}
Objective: to determine the level of blood markers of cellular Human Cluster of Differentiation 147 (CD147) by ELISA assay. Immunological system, establishing the profile in patientsSARSCoV-2 invades host cells via a novel route CD147-spike protein. Methods: a case-control study including 30 patients (10 female) with SARS-CoV-2 repertory disorder whose follow-up was carried out at the outpatient clinic of the intensive care unit Service at Al Hussain Hospital, southern of Iraq, Patients were divided into three subgroups based on the onset of symptoms within the first 6 days of the acute phase of disease subgroup A, subgroup B, and C. according to the type of drugs. Main systemic subgroup. Treatment group included 60 outpatients (25 female) being submitted to follow-up at the same hospital for non-inflammatory diseases. For determined the plasmatic levels of Human Cluster of Differentiation 147 (CD147), groups of S proteins Results: To start with, in vitro antiviral tests showed Meplazumab, infliximab and Etrolizumab an foe of CD147 adapted neutralizer, essentially hindered the infections from attacking host cells in comparison to controls, patients with presented concentrations of CD147 products (determined by plasmatic levels of $S$ protein circulating in blood. Conclusion: our results indicate the presence of molecular SARS-CoV-2 invades respiratory cells determined by assay in patients which confirm a decrease in the defense capacity of the cellular system against toxicity induced by drugs in these patients.
\end{abstract}

